\title{
Provoking the Construction of a Structure for Coordinating $n+1$ Levels of Units
}

\author{
Anderson Norton \\ Virginia Tech \\ $540-231-6942$ \\ norton3@,vt.edu
}

\author{
Steven Boyce \\ Portland State University
}

\begin{abstract}
In considering mathematical development across multiple domains, researchers have implicated the critical role of an individual's ability to produce and coordinate units. Here, we describe a theoretically grounded instructional approach for promoting growth in units coordination. Our approach is informed by neuroscience, as well as existing research on units coordination. We present promising results from implementing our approach in a ten-week teaching experiment with a sixth-grade student named Cody. We demonstrate how, over the course of the 14 teaching sessions, Cody progressed from activity involving the coordination of two levels of units, to activity involving the coordination of three levels of units.
\end{abstract}

Keywords: cognitive psychology; middle school; neo-Piagetian theory, educational neuroscience; units coordination 
Integers, fractions, and algebra present notorious challenges for our students. Recent research on students' mathematical ways of operating indicates a common root cause. Namely, many students lack the ways of operating necessary to produce and coordinate the various levels of units involved in reasoning flexibly within each domain. With fractions, for example, these units include a unit fraction, the whole, and the composite unit formed by taking multiple unit fractions as a single quantity: For example, understanding $7 / 5$ as a quantity (unit) composed of 7 units of 1/5, five of which constitute the whole (Steffe \& Olive, 2010). A growing body of research indicates that the number of levels of units students can coordinate mediates opportunities for learning across several domains of mathematics (Ellis, 2007; Hackenberg, 2013; Hackenberg \& Tillema, 2009; Izsák, Jacobsen, de Araujo, \& Orrill, 2012; Olive \& Çağlayan, 2008; Ulrich, 2012). This finding introduces a serious problem for mathematics educators because, even by middle school, few students can readily coordinate three levels of units (Boyce \& Norton, in review). Our study addresses this problem.

We conducted a teaching experiment to test a theoretically grounded approach to promoting growth in students' abilities to coordinate multiple levels of units. We used existing research on units coordination along with general findings from cognitive neuroscience to design sequences of instructional tasks. What kinds of growth might we promote over the course of the teaching experiment? What constraints might we experience in students' ways of operating during the tasks? This paper reports on results with a sixth-grade student during the ten-week teaching experiment. We begin with an overview of units coordination: what it is; why it is important; and how we might promote it. 


\section{What is Units Coordination?}

From a neo-Piagetian perspective, building models of students' mathematics involves positing psychological structures that explain and predict students' sensorimotor (overtly physical, including verbal) activity when students are engaged in problematic situations (von Glasersfeld \& Steffe, 1991). This observable activity becomes the basis for researchers to make inferences about students' internalized actions and the mental structures that might organize them. Once these internalized actions are organized within a structure for operating, we refer to them as interiorized actions, or operations. In addition to operational schemes (von Glasersfeld, 1995), structures include groupings of operations that organize the ways in which operations can be composed and reversed (Piaget, 1970/1968). For example, children first produce the number 12 as the result of coordinated counting activity. Once their mental actions are organized as operations, 12 can become a composite unit (Steffe, 1992)—part of a structure in which 12 is composed of twelve units of 1 .

Units coordinating refers to students' activity in producing units, including composite units. When this mental activity is interiorized, the actions become organized as operations within units coordinating structures. In particular, Steffe (1992) has described a kind of units coordination wherein a student distributes one composite unit over the elements of a second composite unit. Interiorization of this activity results in a structure of embedded units, as defined by the distributing operation. We use the following task to illustrate the various kinds of units students might produce and the operations they might use to coordinate them within units coordinating structures.

The student is given a short bar, a medium bar, and a long bar and is told that the short bar fits into the medium bar three times and that the medium bar fits into the long bar four times; 
then the student is asked to determine how many times the small bar fits into the long bar (see Figure 1).
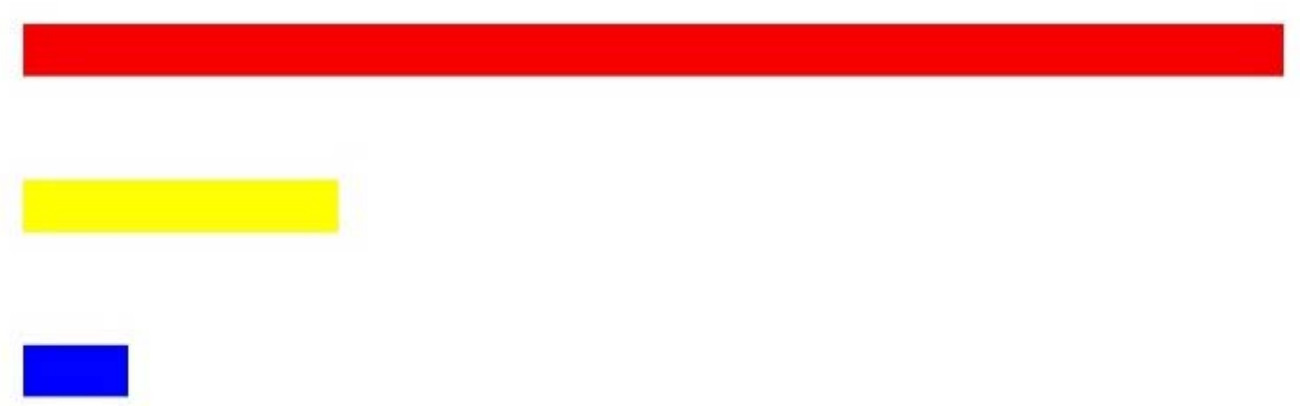

Figure 1. Bars task.

In addition to distributing, relevant operations may include segmenting, by which students mark off equal lengths of a given unit, and iterating, by which students produce a new unit from a number of connected copies of another unit (Steffe, 1992). Researchers use such constructs to explain observed activity and infer how students operate with units. In this paper, we refer to students who have interiorized actions for assimilating with $n$ levels of units as operating at "Stage $n$ " (where $\mathrm{n}=1,2$ or 3 ). Note that assimilating with $n$ levels of units involves the student interpreting a situation as an instance in which she can distinguish $n$ levels of units and organize them within a units coordinating structure; the units and levels do not exist in the situation itself but in how the situation is interpreted. Table 1 outlines ways of operating associated with each level, along with typical responses to the bars task.

Table 1. Stages of Units Coordination

\begin{tabular}{|l|l|l|}
\hline & Students' Unit Structures & Students' Reasoning on the Bars Task \\
\hline Stage & Students can assimilate with & Students use the short bar to mentally segment the \\
\hline
\end{tabular}




\begin{tabular}{|l|l|l|}
\hline $\mathbf{1}$ & $\begin{array}{l}\text { (take as given) one level of } \\
\text { units, and may coordinate two } \\
\text { levels of units in activity. }\end{array}$ & $\begin{array}{l}\text { long bar, imagining how many times it would fit } \\
\text { into the long bar. This activity might be indicated } \\
\text { by head nods or sub-vocal counting. }\end{array}$ \\
\hline $\begin{array}{l}\text { Stage } \\
\mathbf{2}\end{array}$ & $\begin{array}{l}\text { Students can assimilate with } \\
\text { two levels of units (a composite } \\
\text { unit), and may coordinate three } \\
\text { levels of units in activity. }\end{array}$ & $\begin{array}{l}\text { Students mentally iterate the medium bar four } \\
\text { times, with each iteration representing a 3. This } \\
\text { activity might be indicated by the student uttering } \\
\text { "3,3,3, and 3; 12." }\end{array}$ \\
\hline $\begin{array}{l}\text { Stage } \\
\mathbf{3}\end{array}$ & $\begin{array}{l}\text { Students can assimilate with } \\
\text { three levels of units (a } \\
\text { composite unit of composite } \\
\text { units), and can thus flexibly } \\
\text { switch between three-level } \\
\text { structures. }\end{array}$ & $\begin{array}{l}\text { Students immediately understand that there are four } \\
\text { threes in the long bar. This assimilation of the task } \\
\text { might be indicated an immediate response of "12", } \\
\text { buttressed by an argument that 12 is four 3s. }\end{array}$ \\
\hline
\end{tabular}

The three stages in Table 1 represent broad categories for operating, defined by the number of levels of units with which students can assimilate situations and the number of levels they can produce through activity. There is plenty of room for content-related growth within each stage, and researchers have offered finer-grained progressions within specific content domains. For example, Steffe (1992) describes two counting sequences - the initial number sequence and the tacitly-nested number sequence - that fit within Stage 1. Discussion of these sub-stages is beyond the scope of this paper, but interested readers can find an exquisite elaboration by Ulrich (in press). The broad stages defined in Table 1 align with the multiplicative concepts identified by Hackenberg (2010), except we frame them more generally to include content domains, such as integer addition (Ulrich, 2012), that may not involve multiplication.

We expect students to produce and coordinate units through activity first. Once students internalize that activity, they can begin to organize it within an assimilatory structure. Figure 2 illustrates how such operations might be organized. In particular, if a student is operating at Stage 3, she could assimilate 24 as a unit containing eight units of 3. Moreover, she would understand the commutativity of this structure to simultaneously conceive of 24 as three 8 s. Such a structure would enable her to operate flexibly within these units so that if she were tasked with 
determining the number of $3 \mathrm{~s}$ in two $24 \mathrm{~s}$, she would not need to compute the value of the two $24 \mathrm{~s}$; rather, she could solve the task more efficiently by considering the two units of 8 threes that they contain.

a unit of eight units each containing three units

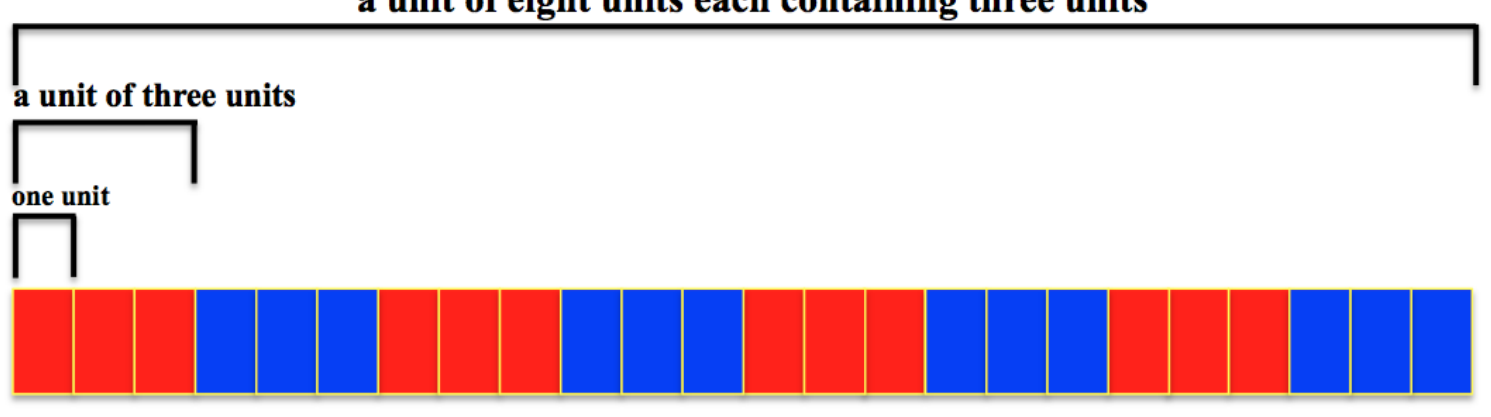

Figure 2. A structure for organizing three levels of units.

\section{Why is Units Coordination Important?}

Because the focus of our study, Cody, was operating at Stage 1 (see Table 1), we focus on the affordances and limitations of the associated ways of operating, as well as some new ways of operating that become available to students like Cody if they progress to Stage 2. These ways of operating cover multiple mathematical domains, including whole number multiplication, fractions knowledge, and algebraic reasoning. In this section, we review research findings on Stage 1 students' ways of operating within each of these three domains.

Units Coordination in Whole Number Multiplication. In order to distinguish students' ways of operating with multiplicative relationships from their rote use of multiplication facts and procedures, multiplicative reasoning has arisen as a key construct in mathematics education (e.g., Fischbein, Deri, Nello, \& Marino, 1985; Kaput, 1989). Composite units are the basic building blocks for multiplicative reasoning (Steffe, 1994): "For a situation to be established as 
multiplicative, it is necessary to at least coordinate two composite units in such a way that one of the composite units is distributed over the other composite unit" (p. 19). Thus, students operating at Stage 1, who lack an interiorized structure for assimilating with composite units, cannot engage in multiplicative reasoning (Hackenberg, 2010). However, they can solve tasks that, from an observer's point of view, might be considered multiplicative.

Consider the case of Zac, a third-grade student operating at Stage 1 (Steffe, 1992). Zac could not assimilate with composite units, in the sense that he did not unitize them as mathematical objects on which to carry out further activity. However, he could build up composite units by segmenting his number sequence. For example, given an array with six rows of three blocks each, he could determine the total number of blocks even when five of the rows were hidden. He did this by employing a "linear spatial pattern" wherein he laid out six segments of three units end-to-end. He kept track of each segment and the three units within each segment by pointing to the (hidden) rows as he counted. As such, he did not build an array of rows and columns, or a unit of units of units. Rather, he built a single unit of eighteen units; those units were still 1s. In other words, consistent with Stage 1, he could coordinate a second level of units in activity but not assimilate with two levels of units or coordinate a third level of units in activity.

Units Coordination with Fractions. Building on the work of the Rational Number Project (Kieren, 1979; Behr, Lesh, Post, \& Silver, 1983), Lamon (2007) endeavored to delineate pedagogical roles for five sub-constructs in students' developing understandings of fractions: part-whole, measurement, ratio, quotient, and operator. After conducting a four-year teaching experiment with five classes of students, beginning in second grade, she concluded that the measurement construct played the most central role in supporting robust conceptions of 
fractions. However, "a more significant factor in overall success was the development of the central multiplicative structures" (Lamon, 2007, p. 660). This finding aligns with Steffe's (2002) reorganization hypothesis and underscores the role of units coordination in students' construction of fractions knowledge.

The reorganization hypothesis posits that "children's fractional schemes can emerge as accommodations in their numerical counting schemes" (Steffe, 2002, p. 267). For students operating at Stage 2, "iterating a unit item and disembedding a numerical part from a numerical whole are two principal operations of the numerical counting scheme" (p. 270). Students operating at Stage 1 have not interiorized these operations because they require simultaneously holding in mind two distinct but related units: Iterating involves maintaining the iterated unit and the composite unit produced through its iteration; and disembedding involves taking a part of a composite whole while maintaining its inclusion in that whole (Steffe \& Olive, 2010). Without the availability of those two ways of operating, Stage 1 students' abilities to construct fractions concepts will remain limited (Hackenberg \& Tillema, 2009). Even part-whole concepts involve the ability to disembed three parts, say, from five equal parts in the whole while maintaining the five-part whole, in order to establish the resulting fraction as three-fifths of the whole. Measurement concepts further require students to treat unit fractions as units they can iterate to produce composite units (Steffe, 2002).

Units Coordination and Algebraic Reasoning. Algebraic reasoning has been characterized as generalized arithmetic (e.g., Blanton \& Kaput, 2005) wherein algebraic symbols and their manipulations serve as proxies for quantities (both known and unknown) and operations on those quantities. Thus, we should expect students' abilities to coordinate unitswhether whole number or fractional units - to influence student success with algebra. Several 
studies affirm that expectation (e.g., Ellis, 2007; Hackenberg, 2013; Olive \& Çağlayan, 2008).

We focus on results from clinical interviews that Hackenberg (2013) conducted with six Stage 1 middle school students, illustrating the limitations such students exhibit when confronting algebraic situations.

In one particularly illustrative example, Hackenberg posed a variant of the studentprofessor problem (cf., Clement, Lochhead, \& Monk, 1981): "Stephen has a cord for his iPod that is some number of feet long. His cord is five times the length of Rebecca's cord. Could you draw a picture of this situation? Can you write an equation for this situation?" (Hackenberg, 2013, p. 552). One of the most sophisticated responses came from a seventh-grade student named Henry. Like most of the other Stage 1 students, at first Henry drew one cord (Stephen's) to be a little more than half of the other cord (Rebecca's). However, he independently corrected his drawing by iterating the length of one cord five times to produce the length of the other cord, and he ultimately sorted out that Stephen's cord was the longer one. When asked for an equation, Henry seemed to reverse the relationship as many students do with the student-professor problem (Kaput \& Clement, 1979) writing an equation that would make Rebecca's cord longer. Although he corrected this reversal, he also altered its value: " $\mathrm{R} \times 4=\mathrm{S}$ tephens cord" (p. 553), arguing that Rebecca already had a cord, so Stephen only needed four more.

Henry's responses typify the limitations of Stage 1 operating in algebraic reasoning. He was able to iterate a unit of 1 in activity (the length of Rebecca's cord), but he had trouble symbolizing the relationship produced through that activity. When he finally settled on an equation, it represented the additive relationship of adding on four more cords, rather than a multiplicative relationship between the two lengths. It seems that Henry could not maintain the relationship of the one unit, Rebecca's cord, embedded within the composite unit, Stephen's 
cord, which resulted from iterating Rebecca's cord five times. We already knew this was a limitation in Stage 1 operating, but Hackenberg (2013) has provided clear evidence of its implications for algebraic reasoning.

\section{How Might We Promote Units Coordination?}

Attempts to provoke growth in students' ways of operating through direct instruction-or any other instructional method that does not explicitly account for the student's current ways of operating - are unlikely to yield desired results (Confrey, 1990; Olive \& Vomvoridi, 2006). Rather, students are more likely to respond to such instruction by modifying their behavior for a given situation, perhaps generating procedural or social schemes that only mask the limitations of their underlying ways of operating (Norton \& D'Ambrosio, 2008). As relatively permanent and generalizable ways of operating, it is the nature of operational structures to resist change until those ways of operating and organizing experience become perpetually frustrated and untenable in the face of new experience (von Glasersfeld, 1995).

With units coordination in particular, there is a distinction between what students can do in activity and the operations they have interiorized as assimilatory structures (Steffe \& Olive, 2010). Students operating at Stage $n$ can assimilate with $n$ levels of units, and can produce $n+1$ levels of units in activity. Promoting higher stages of units coordination amounts to provoking the internalization and then the interiorization of the $n+1$ levels of units that students produce through activity. We turn to neuroscience for clues on how to support such a structuring of mental activity.

Three broad findings from neuroscience studies influence our approach—all concern the neo-cortex (the outer region of the human brain) and particularly the frontal and parietal lobes, 
as depicted in Figure 3. First, physical actions are associated with neural activation in the sensorimotor region - the red and blue areas in Figure 3, which sit at the border of the frontal (orange) and parietal (green) lobes. We say that students have internalized an action when they can imagine performing it without physically carrying it out (cf., Nemirovsky \& Ferrara, 2008). Internalized action is associated with activation in the premotor region of the frontal lobe, just in front of the motor (red) area (Buccino et al., 2001). This region is generally associated with working memory — "the temporary storage and manipulation of information"- —which is supported by imagined actions (Logie, 1995, p. 63). When we refer to the kinds of units students can produce in activity, this includes imagined/internalized action.

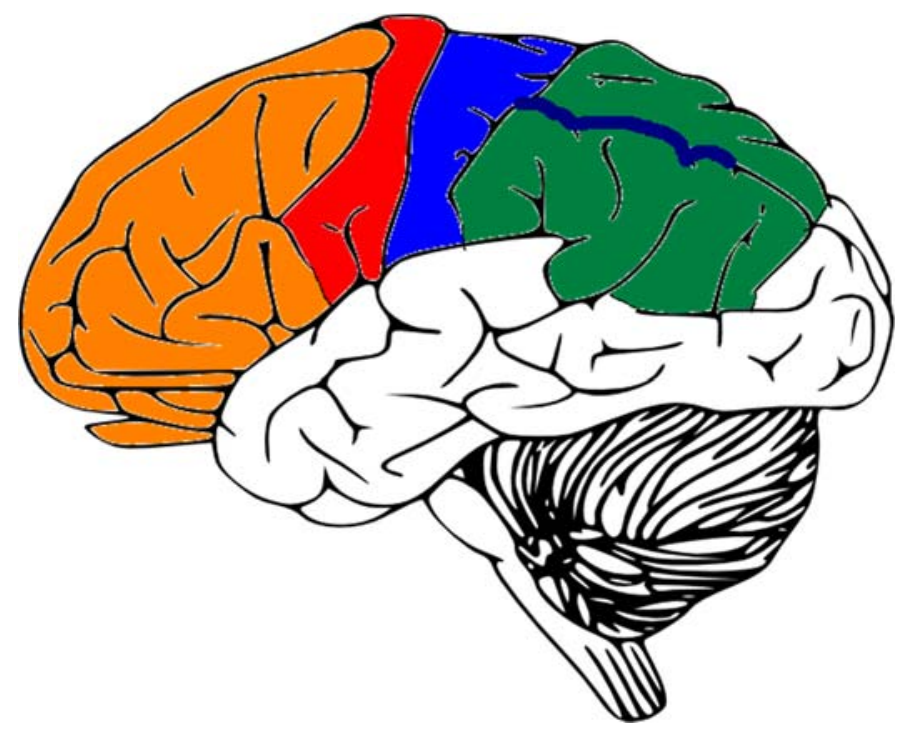

Figure 3. The frontal (orange and red) and parietal lobes (blue, purple and green) of the neocortex.

Second, neuroscience consistently implicates an area within the parietal lobe- the intraparietal sulcus (the purple segment in Figure 3) - for tasks that involve numerical processing, such as counting and comparing quantities (Piazza, Izard, Pinel, Le Bihan, \& 
Dehaene, 2004). More generally, the parietal lobe is associated with objects for action (Buccino et al., 2001), including numbers for computing (Chochon, Cohen, Van De Moortele, \& Dehaene, 1999) and geometric shapes for rotating (Cohen et al., 1996). Because interiorized actions have been organized within a structure for operating, they can be acted upon by other operations, like objects (Pascual-Leone, Johnson, \& Agostino, 2010; Piaget, 1972/1970); so, these findings suggest that we should expect the use of interiorized actions to correlate with neural activity in the parietal lobe.

Third, studies comparing novices and experts consistently correlate mathematical development with a general frontal to parietal shift in neural activity (e.g., Ansari \& Dhital, 2006; Rivera, Reiss, Eckert, \& Menon, 2005). Furthermore, tasks with high cognitive demand tend to evoke greater coherence in (communication between) neural activity across these two lobes (Sauseng, Klimesch, Schabus, Doppelmayr, 2005). In other words, neural activity in the frontal and parietal lobes is synchronized during cognitively demanding tasks.

From a neo-Piagetian perspective, we interpret the above findings as suggesting that the use of units coordination structures should correlate with functioning within the parietal lobe (the intraparietal sulcus, in particular) and that students might coordinate an additional level of units, in activity, by recruiting working memory resources within the frontal lobe. To support the frontal to parietal shift that would correspond to the stage-wise development of units coordinating structures, we would need to engage students in tasks that sustain coherent activity across those two lobes. The idea is that the associated cognitive demand would induce reorganizations within the intraparietal sulcus. In other words, the deliberately controlled activity of coordinating $n+1$ levels of units might eventually become interiorized as the kind of automated activity that characterizes Stage $n+1$. 
Based on this interpretation of neuroscience findings, we designed sequences of tasks for

Cody —a Stage 1 student— that would engage him in coordinating two levels of units, in activity, in multiple ways. These tasks would require him to maintain two-level relationships that he had built through activity (presumably in working memory) as he worked to build new two-level relationships. To support Cody's ability to keep track of and relate the various two-level relationships, we provided Cody with manipulatives to represent the relationships between units. Manipulatives also afford opportunities for students to engage in new activities by providing figurative material to act upon (Clements, 2000). Additionally, we periodically encouraged Cody to reflect on his activity so that he might begin "taking [these] actions as objects of thought" (Wheatley, 1992).

\section{Methods}

Teaching experiments involve intensive and longitudinal interactions between a pair of researchers and one or more students (Steffe \& Thompson, 2000). During each of the teaching sessions in a teaching experiment, the researchers attempt to build, test, and refine a model of the student's thinking and learning. To support this goal, the researchers design tasks and questions intended to provoke mathematical activity, usually through some sort of manipulative as a medium. While one of the researchers is primarily responsible for interacting with the student, the other researcher serves as an observer of those interactions. This allows the research team to reflect on the efficacy of the interactions between sessions; from time to time, the observer might also interject during a session to redirect a task or question. The researchers use video recordings to support retrospective analysis of the teaching sessions, in between sessions and at the end of the teaching experiment. By looking back across all videos, the researchers can determine 
whether their final model of the student's ways of operating consistently explains and predicts the student's activity.

We conducted our teaching experiment with a pair of sixth-grade students, named Cody and Vivian. We selected Cody and Vivian for the teaching experiment after interviewing several students from their class. During our 15-minute interviews with each student, we determined that Cody was operating at Stage 1 and that Vivian was operating at Stage 2. Thus, both students had room to advance in constructing units coordinating structures. However, after the second session, Vivian did not regularly participate in the teaching experiment, so we focus on Cody's development and use Vivian's activity as a contrast to Cody's activity.

\section{Our Approach}

We designed the following framework for generating tasks for Cody during the teaching experiment. Starting with a number, say 30 , its prime factorization determines a maximum number of levels of whole numbers - $m+1$, where $m$ represents the number of prime factors (counting repeats). For instance, $20=2 \times 2 \times 5$ has $3+1=4$ possible levels. We can build these four levels in any context that students can assimilate as an embedding structure. For instance, we can consider a box of cups, where each cup has a specified number of chips and each chip has a specified value.

Level A: cents

Level B: chips, with relation BA: 2 cents per chip

Level C: cups, with relation CB: 5 chips per cup

Level D: boxes, with relation DC: 2 cups per box 
Now we consider all possible permutations of two levels, from among A, B, C, and D. There are $4 \times 3=12$ such permutations, and each of them suggests a task. For example, consider DA: "How much is a box worth?" We can also consider permutations of three levels, where the middle level is an intermediate level. For example, consider ABD: "If you have 14 cents, how many more chips do you need to make a box?"

This approach to task design fits the framework of units coordination and the principles outlined above, regarding how we might promote higher levels of units coordination. First, if a student has interiorized $n$ levels of units, tasks should challenge the student to coordinate $n+1$ units in activity. This means the task setting needs to include at least $n+1$ levels, so that tasks might provoke the internalization and organization of $n+1$ levels. The units students coordinate need to vary within the same problem setting so that students are provoked to simultaneously maintain multiple relationships involving $n+1$ units. Second, tangible media provide students with opportunities to physically enact these various coordinations (Clements, 2000). Finally, students need opportunities to reflect on their activity (Wheatley, 1992; cf., Simon \& Tzur, 2004). The general task setting is amenable to such reflection because it supports physical activity related to packing and unpacking units. Students can re-present these activities within diagrams and other drawings to illustrate relationships between various units.

\section{Data Analysis}

Our teaching experiment began on September 13,2012, during which we affirmed our initial assessment of Cody (from the clinical interview) as a student who had not yet interiorized two levels of units. During the next session, on September 18, we initiated our approach to promoting that development. Twelve more 30-minute teaching sessions followed, charting a progression in Cody's units coordinating activity. All 14 teaching sessions were video recorded 
so that the researchers could meet between sessions to form initial models of Cody's ways of operating and to design tasks for the next session. At the end of the teaching experiment, the 14 teaching sessions were completely transcribed. The researchers recorded notes in the transcription to chart any consistencies, inconsistencies, progressions, and regressions in Cody's ways of operating across sessions and contexts. Thus, in addition to our evolving model of Cody's ways of operating across the 14 teaching sessions, we had a retrospective understanding of how Cody's ways of operating might have evolved. We used this understanding to select relevant episodes to share in this paper.

\section{Results}

In this section, we share our analysis of several key segments within the 14 teaching sessions. Each segment serves one of the following three purposes: to demonstrate how Cody reasoned with units toward the beginning of the teaching experiment; to illustrate our approach to provoke growth in Cody's units coordinating activity; or to demonstrate how Cody's ways of operating with units had changed toward the end of the teaching experiment.

\section{Cody's Initial Ways of Operating with Units}

The purpose of the first three teaching sessions of the teaching experiment was to investigate Cody's available ways of operating with units. Several themes emerged during these sessions. Consistent with Stage 1 (see Table 1), he was able to coordinate two levels of units in activity, but the two-level structures he built took the form of segmented number lines (like Zac) rather than two-dimensional arrays. He built up composite units through repeated addition of the smallest unit and never broke down the larger unit by leveraging a multiplicative relationship. He knew many multiplication facts and relied on those facts to make fortuitous connections to numbers within the problematic situations, rather than to represent an embedded or distributive 
relationship between referent units. He could coordinate across two two-level relationships only when there was figurative material available to bridge them. In the absence of figurative material, he did not succeed in solving problems involving intermediary units, and when he tried, he ended up conflating the intermediary unit with one of the other units. This became especially clear when working with the abstract unit of cents, for which there was no physical referent. We chose the following seven episodes from the first three teaching sessions to substantiate these themes.

Episode 1: September 13. Early in this first teaching session, Cody demonstrated an ability to coordinate two levels of units in activity, but this episode illustrates some of the difficulty Cody had while doing so. The situation involved five cups containing three chips each; Cody and his partner, Vivian (a Stage 2 student), were asked to determine how many more cups they would need to have a total of 24 chips. Cody responded by looking at the five cups in front of him and then saying, "Three, because there's five here; eight times ffffour is... What was the question?" Among many other memorized number facts, Cody knew that 8 times 3 equals 24, so he might have solved the task by seeing that he had five $3 \mathrm{~s}$ and knowing that he needed eight $3 \mathrm{~s}$; thus, he needed three more 3s. As such, he would be building up a three-level structure in activity by relying on number facts and figurative material. Cody's confusion in explaining the solution indicates that he had not built such a structure. Alternatively, he might have taken the five cups of 3 as 15 and counted on three more 3 s to reach 24. This would involve coordinating two levels of units in activity: 15 , and one more 3 is 18 ; two more $3 \mathrm{~s}$ would be 21 , and three more threes would be 24 . 
Episode 2: September 13. A few minutes later in the episode, Cody solved a similar problem in a manner that suggests he had been counting by $3 \mathrm{~s}$ in both episodes. In this and all subsequent transcriptions, 'T:' precedes to the words [and actions] of the teacher-researcher.

T: Close your eyes for a second. [The students comply, and the teacher-researcher removes six cups from the table, leaving a 2-by-3 array of cups, with three chips in each; the teacher-researcher then instructs the students to open their eyes.] Some of your cups have disappeared. I have 18 chips. How many cups should you give me so that I'll have 30 chips?

$\mathrm{V}: 18$ plus 18 is 36 . I think. Yeah.

C: We'd have to have one more cup, because 6 plus 3 [pointing to the cups on the desk] T: Do you all remember the question? Tell me the question so I can make sure you all are answering the one I asked.

V: You have 18 chips. How many more cups would you get until you have 36 .

T: ... until I have 30 chips.

C: [Looks back in two of the cups]

T: It might have sounded like I said 36, but I said 'thirty chips'

C: [Grabs a cup and looks inside; touches a cup; mouths ' 24 '; touches another cup; mouths '27'; wiggles fingers]

C: Four cups. You'd need to get 4 cups to get 30. [pulls four rightmost cups closer to him]

T: Do you agree?

$\mathrm{V}$ : Yep.

T: All right, you explain first, because she explained last time.

C: You used to have 18, and then what I did is I counted up. Because, 21, and then 24,

27, and 30. [touches four cups in synchrony]

T: So you counted up from 18 ?

$\mathrm{C}:$ By $3 \mathrm{~s}$.

T: How many cups will I have altogether, once you give me those?

$\mathrm{C}: 22$.

$\mathrm{T}: 22$ cups altogether?

V: Woah! [Looks over at Cody, smiling]

C: You have 4 cups, plus 18 is 22 . Because 4 and $8 \ldots$

$\mathrm{V}$ : No, he doesn't have 18 cups.

$\mathrm{C}$ : Yes he does.

$\mathrm{V}: 3-18$ chips

Both students seemed confused by the question at first. In Cody's case, it's unclear why he said we needed only one more cup, but " 6 plus 3" likely referred to 18 being 6 times 3 ; he often used the word "plus" when actually referring to multiplication, presumably because, for him, multiplication was repeated addition. Eventually, Cody solved the problem by counting on 
from 18 by $3 \mathrm{~s}$. However, he conflated the units of cups and chips, claiming that we would have 18 plus 4 cups! This became a theme in Cody's ways of operating throughout the first three sessions.

Episode 3: September 13. The disconnect between Cody's use of multiplication and his production of two-dimensional arrays became apparent at the end of the first session. The teacher-researcher had asked the students to form as many rectangular arrangements as they could, to form a total of 32 desks (represented by chips). Vivian seemed to anticipate several arrangements before confirming them with the chips, and she also recognized commutative pairs (e.g., two rows of 16 and 16 rows of 2 ). In contrast, Cody did not generate a single novel solution, but rather, justified Vivian's solutions with post-hoc explanations, using his multiplication facts (e.g., 8 rows of 4 worked "because 8 times 4 is 32 ").

Episode 4: September 18. The teacher-researcher began this second session by asking the students how many 4s were in two 12s. Vivian had responded "3" at first, and Cody corrected her by noting their were two 12s, which was 24 . When Vivian corrected her response, "6", Cody replied, "It could be 6, or 8 three times, because 8 times 3 is 24 too." Cody's actions in this episode indicate that he was not comfortable working with a unit of units: Rather than working with two 12 s, he computed the result, 24 , and used familiar multiplication facts to offer potential solutions. Although he admitted Vivian could be right, his alternative solution was disconnected from the referent units of 4 and 12 .

Episode 5: September 18. Later in the session, the teacher-researcher asked Cody to put three chips in each of five cups, to put these five cups in a box, and to close the box. Cody's response illustrates another propensity in his ways of operating with units.

T: If the chip is worth 2 cents, how much money would be in the box? 
V: [After 8 seconds of both students silently concentrating] I don't know how to figure that out. Because I know there's 15 chips in here [points to box].

C: Ooh!

V: Oh wait, no. Never mind.

C: I know what it is, can I say it?

T: Hold on. You [Vivian] got it now too?

V: No.

T: All right, but you had an idea. Cody, what do you think?

C: Since these are 2 [points to chips], these chips are 2, and there's 5. Since there's 6 in each one, there's $2,4,6$,

T: Okay

$\mathrm{V}$ : Oh, okay, I just got it now.

C: I put these 5 cups, and it equals 6 times 5 equals 30 [smiles].

Cody solved the problem by building up- " $2,4,6$ "-from the smallest unit (cents), and then considering the value for five of these collections. Vivian subsequently solved the problem by determining the number of chips in the box ( 5 times 3 ) and then multiplying this result by 2 . Despite repeated prodding from the teacher-researcher and repeated explanations from Vivian, Cody could not make sense of this approach, presumably because it involved taking a composite unit-15 $2 \mathrm{~s}-$ for granted, rather than building it up through activity. Thus, Cody's actions in this episode fit our initial assessment that he could not organize two levels of units, except by building them up through activity.

Episode 6: September 18. At the end of the session, the teacher-researcher asked Cody and Vivian to draw pictures that would help them represent the various relationships between the cents, chips, cups, and box. Figure 4 displays Cody's drawing.

Figure 4. Cody's first drawing.

T: Why did you go for smiley faces? 
C: Because I like smiley faces

T: Does your picture help us figure out how many chips are in each cup?

V: Actually, I just realized - it does! Two eyes and a smiley face.

T: I was wondering if he did that on purpose. Do you think he did that on purpose?

V: No.

T: Did you do it on purpose, Cody [chuckling]?

C: [Shakes head no, smiling]

T: Does your picture help us figure out how much 1 chip is worth?

C: Yeah, because there's 1 chip there, 2, 3 [pointing to eyes and mouth within a single smiley face]

T: That tells us how many chips are in 1 cup. Does your picture help us figure out how much money 1 chip is worth?

C: Yes, 2 cents [points to where he has written ' $=2 \mathrm{c}$ ' to right of the rectangle]. That was neat.

T: Did you have a comment on Cody's?

V: You need to write - that means the whole thing is equal to 2 cents, the way it looks.

T: It might confuse somebody.

V: What equals 2 cents? The whole thing? That's what it looks like.

C: [Draws to left of ' $=$ '] This little smiley face.

In his drawing, Cody had attended to two levels of units only - the five cups in the box, which constituted a unit of units. When he was prompted to attend to other units, Cody could not disambiguate their relationships, especially when working with the abstract unit of cents. At first, he attributed two cents to the entire box. When questioned about this the first time, he seemed to conflate the relationship between cents and chips with the relationship between chips and cups ("1 chip there, 2, 3"). When questioned a second time he seemed to attribute two cents to the entire smiley face, representing a cup or three chips.

Episode 7: September 25. We began the third session with the same set up as before-a box containing five cups, each containing three chips. The following protocol pertains to that context and provides further indication of Cody's ways of operating with units. In particular, it illustrates his propensity for building up composite units, through activity, from the smallest unit, and his reliance on known multiplication facts, often with little reference to the situation. 
T: This is what all boxes are like. How many of these boxes would I need to have 60 chips?

C: Can I use paper?

T: Tell me what you would do, and then I'll help you.

C: What I would do, is since there's... I would do 5 times 3 ,

T: Okay.

C: 15 plus 15 plus 15 and see what that is. And whether that answer is, if it's not 60 , then I'll try to get closer.

T: Okay. So what do you... Tell me what you want to know. 15 plus 15, plus 15 ?

C: Yeah.

T: All right. Do you know about what it is?

C: 45 .

T: 45. Okay.

C: [Concentrating, looking at ceiling silently for several seconds] You'd have four boxes [smiles]

T: How'd you figure that out?

C: Because, 5 times 3,15 , and then 15 plus 15 is 30 . Plus 1 more is 45 , and that's 3 . Then what I did is plussed 45 plus 15 [gesture putting hand down] and 5 times $5-5$ plus 5 is 10. So what I did is I carried the one, put it over the 4, and then 4 times 2 equals 6 .

T: You did it all in your head.

C: Yep.

T: You didn't need paper.

C: Nope.

T: It's good to practice them in your head, don't you think? If you practice them in your head, it gets easier, right?

C: Uhum.

T: Okay. So, do you remember what my question was?

C: How much boxes would you need if you had 60 chips.

T: Okay. How many cups would I need to have 60 chips?

C: Chips?

T: Uh hum. How many cups would I need to have 60 chips?

$\mathrm{C}$ : [Counting on fingers, raising 12 fingers in sequence on left, right, left hands; concentrates for 1 second after last finger raise] 12 !

T: How did you figure that out?

C: Counting by $5 \mathrm{~s}$, see? [Simultaneously says 5 number sequence while raising fingers]

T: Okay. Why did you count by $5 \mathrm{~s}$ ?

C: Because, if I had counted by... [pauses 3 seconds] I couldn't count by 3s, I couldn't count by $4 \mathrm{~s}$, I couldn't count by $6 \mathrm{~s}$ or $7 \mathrm{~s}$, so I did $5 \mathrm{~s}$.

T: You chose 5 because you're good at counting by $5 \mathrm{~s}$, or...

C: Yes.

In responding to the first question — the number of boxes needed for 60 chips - Cody successfully solved the task by building up from three chips per cup to 15 chips per box (five 
cups). This involved coordinating two levels of units in activity, but once he determined there were 15 chips per box, he could treat that value as a single unit to be coordinated with the number of boxes needed to make 60 chips; in other words, he only needed to work with two levels of units at a time. In responding to the second question - the number of cups needed to make 60 chips - we might expect students to use the relation of five cups per box to break down the four boxes into 4 times 5 cups. Alternatively, students might determine how many $3 \mathrm{~s}$ (the number of chips per cup) are in 60. However, Cody had not interiorized two levels of units and, therefore, he did not assimilate with composite units, such as four $5 \mathrm{~s}$; and he could not produce the three-level structure necessary for bridging chips, cups, and boxes. So, he attempted to build up the 60 chips through double counting and determined that there were twelve $5 \mathrm{~s}$ in 60 . In doing so, he had conflated the relationship between chips and cups with the relationship between cups and boxes.

Summary. Collectively, these first seven episodes indicate the affordances and limitations of Cody's ways of operating with units. He could produce two-level structures through activity, by building up from the smallest unit. However, when the task required him to either assimilate with a two-level structure, or build up a three-level structure through activity, he had to rely on figurative material. Else, he would conflate units or simply rely upon known multiplication facts with no reference to the units contextualized in the problematic situation.

\section{Illustrating Our Approach}

Our main goal in working with Cody was to provoke the interiorization of a two-level structure and to provoke the production of three levels of units in activity. In other words, we attempted to support Cody's development from Stage 1 to Stage 2 (see Table 1). Our approach is characterized by the use of a context with three or more levels of embedded relationships and a 
rapid succession of questioning involving various ways in which the units are embedded within one another. We hypothesized that, as students attempted to simultaneously hold these various two-level relationships in mind, the cognitive demand would induce new interiorized structures for organizing them.

Episode 8: September 27. The following protocol, which occurred during the fourth teaching session, illustrates our approach. The context of the questioning involved a box with three cups, each containing two chips, each worth two cents.

T: Okay, so you think you're ready.

C: Yeah.

T: What is the box worth?

C: That box [looking straight ahead] 2, 4, 6-12.

T: You went, '2, 4, 6' and then you jumped to '12.' How'd you do that?

C: Since each chip's worth two, and there's three, and 3 times 2 equals 6 , and then I plussed 6 plus 6 since there's 2 cups and that equals 12 .

T: Nice! I like the way you explained that.

C: Thank you.

T: What's a cup worth?

C: A cup, cup. What's a cup worth [looks down at his paper, then back up at T]. Six.

T: How'd you do that?

C: Three. Since there's 3 chips, and each one equals 2, 3 times 2, equals 6 .

$\mathrm{T}$ : I really like the way you're explaining. How many chips are there?

C: Chips. There's [looks at paper and then off to side]. Six.

T: Nice.

C: Thank you.

To this point, Cody had been asked about three different two-level relationships: box to cents, cups to cents, and box to chips. These are the only three two-level relationships with an intermediary unit. Note that, as before, Cody began by building up the higher-level unit from the smallest unit ('2, 4, 6' and then ' 6 plus 6'). However, once he had built up the relationship between cents and cups (' $2,4,6$ ') in determining what a box was worth, he was able to break it down when determining what a cup was worth: "Since there's 3 chips, and each one equals 2, 3 times 2, equals 6." Thus, this segment affirms our model of Cody's current ways of operating 
with units and indicates how our approach might provoke growth in those ways of operating. Namely, because Cody still had in mind the composite unit he had just built up (a cup as three $2 \mathrm{~s})$, he was able to work in reverse immediately afterward, when he asked to break it down. Such activity might support his construction of an interiorized composite unit.

Episode 9: September 27. Another aspect of our approach was to encourage Cody to reflect on his activity and the structures he had built up through activity. As part of that effort, the teacher-researcher periodically asked Cody to represent the units and their relationships within a particular context. Figure 5 shows the drawing Cody produced in the middle of the fifth teaching session, to which the following protocol refers.

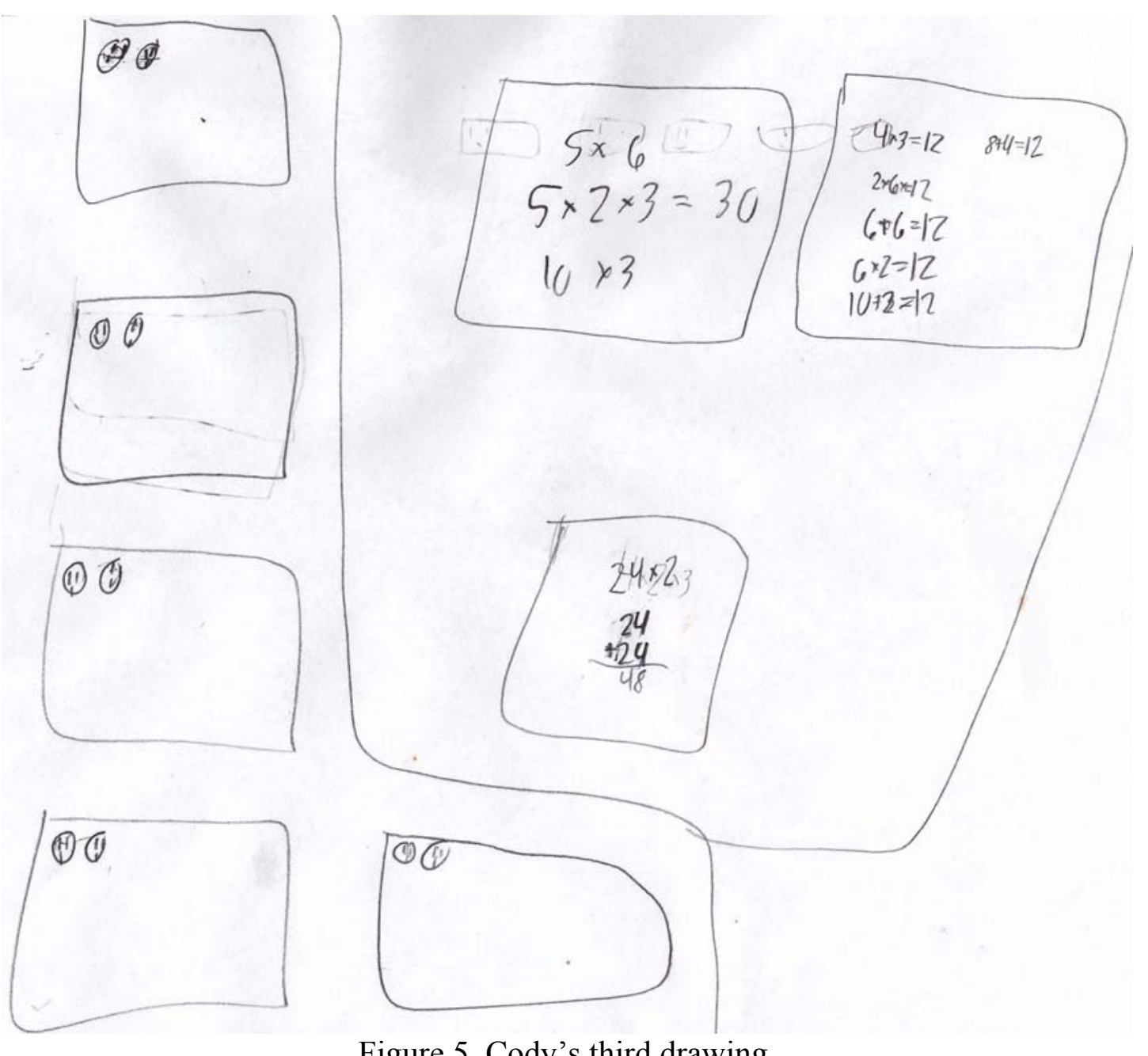

Figure 5. Cody's third drawing. 
T: Now, I'm going to ask you three more questions, and be thinking about how pictures can help you. What if I had 24 chips? How many boxes would I have?

C: [Yawns. Points at individual chips with pencil from top to bottom, left to right, while humming rhythmically. After moving pencil over the chips in the fourth box, stops] Four.

T: How many cups would I have?

C: [Looking at the teacher-researcher, not at his paper] Six.

T: How do you know?

C: [Referring to paper] I counted up, and there's 24 [gestures to column of four boxes] chips in there, and there's four boxes, and there's chips. There's 3. There you go [raises arms as if finished]. Boom!

T: How'd you get 6 ? That didn't look like 6 to me.

C: [Pointing to chips in the top box] 1, 2, 3, 4, 5, 6

T: Oh, there's 6 chips in each box. But what I asked is, how many cups would there be if I had 24 chips?

C: 6. See, 2, 4, 6 [moving pencil from top to bottom] Wait. There would be 8 .

T: 8. Good. Okay. All right. One more question. How much money would I have?

$\mathrm{C}$ : [Appears to count by $2 \mathrm{~s}$ subvocally in sync with pointing at individual chips from top to bottom, left to right. When gets to last box] Wait. Total? [After the teacher-researcher affirms, repeats skip-counting by 2s from beginning] 50 .

T: I think you counted 2 too many. Let me see how you can figure this out. Is there a multiplication problem you can do? 50 is close, but it's off by just a little bit. Can you do a multiplication problem that would make sure you'd get the right answer?

C: [Writes ' $4 \times 2 \times 3$ ', then ' 8 ' centered below the ' $4 \times 2$ '] That would be, 24 ?

T: That equals 24 , but what does the 4 stand for [pointing at top of writing]?

$\mathrm{C}$ : Boxes.

T: What does the '2' stand for?

C: Cups.

T: What does the ' 3 ' stand for?

C: Chips.

T: Now I need money.

$\mathrm{C}$ : That would be how much the...

T: So you have 24 chips, but now we know that each chip is worth 2 cents.

C: [Pointing at chips in top box] Two, ... [interrupted]

T: I know you can do that - you almost got it last time. It's really close to 50. That's what you did last time. I'm wondering if you could do the multiplication for the money, so that you don't have to add, or count by 2 s. Is there something you can do?

C: [After silently focusing inward for about two seconds] No.

In answering this new sequence of questions, Cody relied heavily on the figurative material provided in his drawing. Although he still conflated some of the relationships between the units (e.g., confusing the six chips in each box with the number of cups in four boxes), his drawing seemed to help him keep track of referent units — all except for the number of cents per 
chip. In fact, in several instances he conflated the number of chips with the number of cents;

once, when counting chips during the sixth session, he double counted and justified the result by saying, "they're each equal two." Following that session, we decided that we should replace the abstract unit of cents with a higher-level unit that Cody could more easily represent with his drawings.

Episode 10: October 9. At the beginning of this sixth session, the teacher-researcher introduced a new context. Cody was instructed to place three chips in each of four cups, within each of two boxes. Then he was told that these two boxes made a cart, as represented by their placement on a rolling chair.

T: All right. How many chips are in a cart?

C: [Points at bottom box] There's... How much are they worth?

T: I'm not saying how much they're worth. We're not doing money this time.

C: [Pointing at bottom box, appears to make 4 pointing acts] So [looks up] 3, 3 - that would be 12 . Twenty-four.

T: Woah! How'd you do that?

C: 12 times 12 . Because there's 4 in each one [gestures linearly from left to right on bottom box]. There's four cups [gestures linearly from left to right on bottom box again], three chips in each one [gestures vertically from top to bottom on bottom box], so 4 times 3 is 12 , and 12 plus 12 equals 24 .

$\mathrm{T}$ : Nice! How many chips are in a box?

C: 4. Wait [looks to upper-right]

T: How many chips are in a box?

C: 12 .

T: How'd you know that?

C: How many chips are in box? Because 24 is both, so you minus that, so it would be 12 . T: Nice. So you knew how many were in the cart, and you used that to figure how many were in a box?

C: Yeah. I think this jacket makes me look cooler and smarter.

T: Uh, maybe. But answering these questions is going to make you feel really smart.

C: I feel really smart now, so I'm wearing my jacket.

T: All right. If it makes you feel smarter, maybe it will help you with these questions. How many cups are in a cart?

C: [Looks over at cart] 8.

T: How'd you do that?

C: 4 plus 4

T: I like it! All right. If you have 48 chips, how many boxes would you have?

C: [Looks to up] 2. Wait -4 . 
T: [Laughs]

C: Yeah! [Smiles]

T: Wow you're getting fast! That jacket is making you smarter.

W: [Laughs]

C: I'm going to tell my mom that.

T: All right. If you had 48 chips, how many cups would you have?

C: 48 chips. [Looks up, then over at cart] 16.

T: How'd you do that?

C: 4 - cause 48 , and then that would be 4 boxes. Wait. What was the question? How much...

T: If you have 48 chips, how many cups would you have?

C: There's 12. [looks up] Sixteen.

T: How'd you do that?

C: Because in each one [touches bottom box of cart] there's 8.8 plus 8 equals 16 .

T: Ahh. So you knew that there were two carts, and you used that to figure out the cups.

C: Yep!

Cody operated much as he had before—building from the smallest unit—but the figurative material enabled him to work through intermediary units. For example, when asked how many chips were in a cart, he could imagine the four cups within a box and count by $3 \mathrm{~s}$ to determine the number of chips per box. From there, he could attribute 12 chips to each box in front of him, thus identifying 24 chips in the cart. Moreover, the figurative material enabled him to reflect on his actions in justifying his answer: "There's four cups [gestures linearly from left to right on bottom box again], three chips in each one [gestures vertically from top to bottom on bottom box], so 4 times 3 is 12 , and 12 plus 12 equals 24 ." Upon reflection, he no longer needed to count by $3 \mathrm{~s}$, but rather used multiplication to indicate the relationship between the four cups and 12 chips per box. And, although he confused chips and cups at first, he subsequently used this production to determine how many chips were in a box. Cody continued using results from one production to determine new results: four boxes for 48 chips because there are 24 chips in each of two carts, and there are two boxes per cart; eight cups for 48 chips because there are two carts, and there are eight cups per cart. 
Summary. Episodes 8, 9, and 10 illustrate our approach to provoking growth in Cody's units coordinating activity. Similar episodes occurred within each of the remaining sessions, with some variance in context. Although most of these episodes used arrangements of chips, cups, and boxes, a few involved the use of base-10 blocks or measuring cups. Here, we have provided some indication of the effectiveness of our approach: Cody's retention of unit relationships during rapid sequences of questions and his reliance on figurative material to explicitly maintain the embeddedness of multiple levels of units. In the next section, we will provide evidence for permanent changes in Cody's ways of operating with units.

\section{Demonstrations of Growth}

The purpose of the eleventh teaching session was to continue our approach to provoking new units coordinating activity. During that session, it became apparent that Cody was already developing new ways of operating. Signs of progress included Cody using multiplication with explicit reference to contextualized units, attempts to simultaneously coordinate three levels of units, and reflections on his units coordinating activity. All three were evident during the eleventh teaching session (Episode 11), but the most profound evidence of change occurred during the twelfth teaching session (Episode 12), which followed a three-week period of rest.

Episode 11: October 25. We began the eleventh session with Cody choosing a new chipcup-box-cart structure. He placed two chips in each of six cups, within a single box. He then placed two boxes, as a cart, on a rolling chair, which the teacher-researcher rolled to the other side of the room.

T: How many chips are in each box?

C: [Taps on desk, then splays out five fingers on right hand, one finger at a time on left hand, thumb on right hand, and then forms fist with right hand] 12 .

$\mathrm{T}$ : Is there a multiplication problem you could have done to get that answer? C: 6 times 2 .

T: What's the 6 and what's the 2 ? 
C: How much cups and how much chips.

T: How many chips are in each cart?

C: [Looks at ceiling] 24.

T: How did you do that?

C: 12 times 12 .

T: 12 times 12 equals 24 ?

C: Yes, since there's 12 in each one, and there's 2 [looks back at cart] boxes, that's 24 .

$\mathrm{T}: 12$ times 12 ?

C: 12 times 2.

T: Oh, ok. You said 12 times 12 at first. But you meant 12 plus 12, or 12 times 2 .

C: 12 times 2.

T: Okay. How many cups are in each cart? [Quickly Repeats]

C: [Immediately] 12.

T: How did you know that?

C: Because first, in each one there's 6 . So 6 plus 6 would be 12 - or 6 times 2 .

T: How many chips are in each box?

C: [Immediately] 12.

$\mathrm{T}$ : How many cups are in each cart?

C: How many cups are in each cart. [looks to right corner of room, then back at cart]

Twe- [places totality of fingers on desk at once] Twelve.

T: How many chips are in each cart?

C: How many chips in each cart? [Looking to right corner of room] 24.

T: How many cups are in each box?

C: Six [immediately].

$\mathrm{T}$ : How many chips are in each box?

C: 12 [immediately].

$\mathrm{T}$ : How many cups are in each cart?

C: [Looks straight ahead] 12.

T: How are you getting those answers?

C: Of the stuff that's in there. There's 6 cups in each box, and there's 2 chips in each one, and there's 12 total, with both of them, and there's 24 total with all the chips.

T: If you had 24 cups, how many carts would you have?

C: 24 cups. 1.

T: 24 cups

C: Wait -2 .

T: Two. How do you do that?

C: Since there's 12 in each one, how many cups - [looking at corner of room] there'd be 6 in each one [makes hand motion] that's 12 is one cart [points in direction of cart]. 22.

Cause 12 times 2 is $12-24$ !

T: If you had 3 cups, how many more cups would you need to make a cart?

C: If I had - how much cups?

T: 12 cups - I mean, 3 cups. How many cups would you need to make a cart?

C: 3 cups?

T: How do you figure that?

C: [Yawns] You said 3 cups, how many more carts do I need?

T: If you have 3 cups, how many more cups do you need to make a cart? 
C: [10 seconds, looking straight ahead or at corner of room $] 9$.

T: How'd you figure that?

C: Well, since there's 3 and then 10,11, 12 [holds up thumb and then first and second fingers]. Nine plus 3 equals 12 .

T: Nice. If you had 50 cups, how many chips would you have?

C: [To self, quietly] 50 cups, how many chips would you have. 50 cups [looking at corner of room] Gosh! That's going to be hard [looks over at cart]. So 50 cups - chips. The closest you could get to 50 would be 48 .

Note that Cody answered the first question by building from the smallest unit again, counting by $1 \mathrm{~s}$ on his fingers. However, once he had done this, he was able to restructure his activity as the multiplication of two chips within each of six cups. When asked how he was getting his answers, Cody provided indication that he had internalized three levels of units based on his preceding activity ("There's 6 cups in each box, and there's 2 chips in each one, and there's 12 total, with both of them, and there's 24 total with all the chips"). Although maintaining these relationships likely relied on the figurative material he had used in building up the cart, he no longer needed to manipulate or even look into the boxes. And while Cody still made some of the same conflations that were characteristic of his reasoning in earlier sessions, he reflected on his units coordination activity so that he could quickly correct them. For example, when asked how many carts 24 cups would make, he said " 1 " at first (possibly conflating cups and chips) but then quickly revised his response to "2." On the other hand, he was still not breaking down composite units when their numbers exceeded the figurative material. In particular, when asked how many chips went with 50 cups, he seemed to assimilate the task as a request for the number of carts 50 cups would make, rather than attributing two chips to each of the 50 cups. This persistence of this issue, and some signs of progress, became evident as the episode continued.

T: How many chips would you have if you had 6 cups? 
C: How many chips would you have if you have 6 cups [to self, looks at blank part of desk]. 12.

T: How do you know that?

C: Since there's 2 in each one [gestures to $(1,2)$ cart], 6 times 2 equals 12.

T: How many chips would you have if you had 60 cups?

$\mathrm{C}$ : How many cu? [looks unsure, then smiles, and laughs quietly] A hundred twenty. $\cdots$

T: If I told you the number of cups, what could you do with the number of cups to figure out the number of chips?

C: [Covers face with hands] Hmm. Divide. I'm thinking of dividing [covering mouth with hands; 5 second pause]. So I think divide.

T: Okay. So, for example, if I told you that there were a thousand cups-you don't have to actually compute it.

C: Ooh-ooh. I know the answer.

$\mathrm{T}$ : Tell me what you were going to do

C: I would, since there's a hundred twenty, if there's 60,600 would be [looking at right

corner] one thousand two hundred. And then a thousand - six thousand would be 12 thousand [beaming].

...

T: If you had the thousand cups in front of you, how many chips would there be?

C: $2,4,6,8$ [gestures pointing act with pencil]

$\mathrm{T}$ : Ahh, you could just do that a thousand times, right?

C: Yes.

$\mathrm{T}$ : Is there a multiplication problem you could do?

C: A thousand times 2 .

$\mathrm{T}$ : Would you get the right answer that way?

C: Probably.

Once again, Cody seemed confortable breaking down composite units when he could reference figurative material or when he could perform repeated addition, and now he seemed to be generalizing the two-to-one relationship between chips and cups, but only through powers of 10 , as if he were treating $10 \mathrm{~s}, 100 \mathrm{~s}$, and $1,000 \mathrm{~s}$ as units of 1 . When pressed for the number of chips to accompany 1,000 chips, Cody needed to count by $2 \mathrm{~s}$ before making a tenuous connection to multiplication.

Episode 12: November 15. Due to teacher workdays and Thanksgiving break, there were no teaching sessions between October $23^{\text {rd }}$ and November $15^{\text {th }}$, on which the twelfth session took place. The session began with the teacher-researcher instructing Cody to place ten chips in each 
of five cups, within a single box. Cody placed the box on the cart (rolling chair) along with another, empty box, which was supposd to contain the same arrangements of cups and chips. Before the teacher-researcher began asking questions, Cody spontaneously wrote down the following information: "10 cups, 100 chips, 2 boxes, 1 cart." He subsequently wrote "and 5 cups" and "and 50 chips," next to "10 cups" and "100 chips," respectively. After some simpler questions about the number of chips/cups in a box/cart, the following exchange began.

T: If you have 400 chips...

C: [Gasps, wide-eyed; looks down at paper]

T: If you had 400 chips, how many carts would you have?

C: 400? [Looks at cart] chips. [Gets out of seat] There's a hundred. Two hundred, 300, 400 [walks to right, gesturing picking up/putting down carts as saying number words] Four!

T: Okay. If you had 400 chips, how many boxes would you have?

C: It would be 4 [walks distance he just traversed a moment before from left to right. Stops. So each one [crosses arms]. Eight.

T: Nice. How many cups would you have?

C: [Remaining standing] There's 10 [standing over cart] 20 [moves a step to right, gestures 'putting down,' continues this pattern twice more] 30, 40.

T: All right.

C: 40 .

T: I like the way you did that. You just figured out a lot of stuff.

$\mathrm{C}$ : I think is actually my lucky jacket.

T: Do you think you're doing better on these problems than you did last time when we gave you these kinds of problems, or do you think you're doing about the same, or? C: I think it's better, because I think this jacket makes me smarter [begins playing with spare chips on desk].

T: All right. Okay, I have one more round... What if you have 30 cups. If you have 30 cups, how many carts would you have?

C: [Turns around to face cart] 30 cups [looking up at ceiling]. [Walks in circle around the cart, repeating task] So there's 10 [taps bottom box, starts walking in same direction as before]. Three!

T: If you had 30 cups, how many boxes would you have?

C: Huh?

T: If you had 30 cups, how many boxes would you have?

C: 30 cups, how many boxes would you have. 10 [gesturing with two hands to the cart] 20,30 [hopping to his left]. [Gestures three hand movements from right to left in the same direction, at chest height] Six.

T: If you had 30 cups, how many chips would you have?

C: How much, 30 cups? [Scratches head, walks in circle about cart again] 30 cups - so

that would be three carts. [Looking at right corner of room] How many cups would I have 
- how much chips would I have? [Places hand on top of top box] 10. [Gestures two similar hand movements from left to right] 20,30. No. No. There's 10 - there's 50, 100 [standing in front of cart, but looking to his right. Hops to right] 200. [Hops to right again] 300 !

T: Nice! I like the way you did it.

C: Woo!

T: If you had 50 chips

C: It's the magic jacket.

T: If you had 50 chips, how many more chips would you need to make a cart?

C: Huh?

T: If you had 50 chips, how many more chips would you need to make a cart?

C: [Yawns] 50. [Lays head on arm on top of cart]

T: If you had 30 chips, how many more cups would you need to make a cart?

C: 30 cups?

T: If you had 30 chips -or - did I say cups? If you had 30 chips, how many more cups would you need to make a cart?

C: [Rolling cart from side to side while leaning on it, looking to his right] That would be - how much cups.

T: Yeah, if you had 30 chips, how many cups would you need to make a cart.

C: [Looking straight ahead while resting on the top box] 4 and 6, because -wait. 4 is you said 30 , so that's 3 cups. Then, 5 plus 2, [looking to right corner] because there's 5 here [taps bottom box] and 2 here [gestures toward top box] that's 7 .

T: If you have 5 cups, how many more boxes would you need to make a cart?

C: 5 cups [looking at $\mathrm{T}]$ ?

T: Yeah, if you had 5 cups, how many more boxes would you need to make a cart?

C: [Looks straight ahead while resting head on arm on top box] Boxes? 1.

T: If you have 8 cups, how many more chips would you need to make a cart?

C: If I had 8 cups [looking at T]... Could you say it again please?

T: If you had 8 cups, how many more chips would you need to make a cart?

C: [Looks straight ahead for 4 seconds] That's 80 . Chips or cups?

T: If you have 8 cups, how many more chips would you need to make a cart?

C: 20 .

T: If you had 1 box, how many more chips would you need to make a cart?

C: How many boxes?

T: If you had 1 box, how many more chips would you need to make a cart?

C: 50

T: If you had 1 box, how many more cups would you need to make a cart?

C: [Looks to left for less than a second] 5.

T: That is your smart jacket.

W: That was incredible, Cody.

C: This has to be my smart jacket!

At the start of the teacher session, Cody had written down "100 chips" and " 1 cart", indicating the relationship between those two units. He relied on this relationship to answer the 
first question: Beginning with the physical cart in front of him, Cody counted by 100 s, imagining a new cart for the second, third, and fourth 100 hundred. As such, he coordinated two levels of units in activity: 1s and 100s (carts and chips). Furthermore, he used the result of this coordination to produce a new two-level relationship in activity, when he determined that 400 chips would require 2 times four boxes (two boxes for each of the four carts), using the number of carts as an intermediary unit. He operated similarly with the cups and boxes tasks, once again using the number of carts as an intermediary unit. The latter half of the segment demonstrated that Cody could now work through intermediary units to build three levels of units in activity.

Cody was asked to determine the number of additional cups needed to complete a cart, given 30 chips. Although he needed the teacher-researcher to repeat the task a couple of times and he referred to the physical box in front of him to maintain the unit of five cups per box, he eventually formed the goal of converting chips to cups in order to complete the two sets of five cups in a cart: " 5 plus 2 , because there's 5 here and 2 here." This response provides evidence that Cody could coordinate three levels of units via internalized activity, characteristic of Stage 2. During the subsequent task, he operated similarly and was able to reverse the relationship between cups and chips to produce 80 chips from 8 cups.

Episodes 13a and 13b: October 18 and November 20. As a test of Cody's growth and its generalization across contexts, the researchers decided to reengage Cody in problematic situations similar to ones in which he had engaged leading up to the Episodes 11 and 12. The first of these situations involved the use of base-10 blocks. The protocol shown below (Episode 13a) documents Cody's engagement in the situation during the $10^{\text {th }}$ teaching session. We contrast that activity with Cody's engagement in a similar context during the $13^{\text {th }}$ teaching session 
(Episode 13b). These were the only two sessions in which Cody worked with base-10 blocks, though he had used them in class.

At the start of the teaching session (Episode 13a), Cody had expressed his familiarity in working with base-10 blocks, and at the beginning of the session, the teacher-researcher posed a series of questions about the number of times each manipulative fit into another. The following protocol begins with the teacher-researcher repeating those questions. Throughout the protocol, "single" refers to a $1 \mathrm{~cm}^{3}$ block; "long" refers to a rod made up of 10 linearly-connected singles; "flat" refers to a connected 10-by-10 array of singles; and "cube" refers to a supposedly solid 10by-10-by-10 cube of singles. These were familiar terms for Cody, but he had trouble envisioning the hollow cube as ten flats and instead referred to it as containing 600 singles, presumably because he saw 100 singles in each of the cube's six faces. So, the teacher-researcher encouraged Cody to build his own cube from ten flats.

T: Okay. Let me ask you those questions again, and then I'm going to ask you some new ones. How many singles go into a long?

C: 10 [sliding single from left to right across long on desk].

T: How many longs go into a flat?

$\mathrm{C}$ : [Picks up long and moves it close to cube] 10 [moving the long up the side of the cube].

T: How many singles go into a flat?

$\mathrm{C}: 100$ [moves long from aligned on left side of the cube to aligned in front of the cube].

T: How many singles go into a cube?

C: [Slides long up the front face of the hollow cube] Six.

T: Singles. How many singles go into a cube?

C: [Puts a single on top of the hollow cup] A thousand.

T: Okay. You got all that down. Are you ready for new questions?

C: Yes.

T: Okay. How many longs go into a cube?

C: How many longs. [Holds long vertically on left face of stacked flats] 1, 2 [moving from front to back of the face] 600. [Starts spinning the flats in the middle of the stack] T: Okay. So if you took this apart [the stack] if you took this cube apart into all of its pieces, how many longs would there be?

C: Six. Hundred.

T: Can you show me? Let's take it apart and show me. So this is your cube [puts hand on the stack of 10 flats]. 
C: Ah. Wait - it would be. There's a hundred in each one, and there's 10, so, 100. It would be a thousand.

T: Okay - can you show me?

C: Yes. This would be a hundred [picks up top flat].

$\mathrm{T}$ : Is there a hundred longs in there?

C: Yeah.

T: Show me that there are a hundred longs.

C: There's 10.

T: Oh.

C: But there's a hundred singles?

T: Okay. But I'm asking, how many longs would there be in the whole cube?

C: There would be - I could count by [1 second pause]... What you could do, is you could take it apart.

T: Okay.

C: [Takes off top flat] 10 of these [slides long from left to right along flat] in each one. 10.

T: Okay.

C: $20,30, \ldots 100$ [in sync with re-stacking the flats] A hundred.

Once again, Cody had trouble visualizing the ten 100s in the cube, even after he had constructed a cube from ten flats. At the same time, Cody was conflating singles and longs so that when he was asked how many longs fit into the cube, his initial response was 600 . He only resolved these issues when he physically took apart the ten flats making up the cube, demonstrating a strong reliance on figurative material when coordinating three levels of units: The cube as a unit of 100 units of ten units (a long) of 1 (a single). Even then, he resolution involved building from the smaller unit (longs) in a linear, additive manner: $10,20,30, \ldots 100$. Contrast this way of operating with Cody's response to the same sequence of tasks during the $13^{\text {th }}$ teaching session (Episode $13 b$ ).

T: [Places a single and a long on Cody's desk] How many ti - how many of these [singles] fit into here [long]?

C: 10 .

T: How many of those [longs] fit into here [places flat on Cody's desk]

C: [Yawns] 10. [Afterward gestures sliding the long, aligned horizontally, up the flat.]

T: How many of those [flats] fit into there [places cube on Cody's desk].

C: Do you mean, like that [gestures sliding flat from front to back].

$\mathrm{T}$ : If this was... 
C: 10 .

T: ...solid. Okay. So we're pretending all these things are solid, right? Okay.

[Interruption by announcement] All right, ready? How many of the singles fit into the longs?

$\mathrm{C}: 10$.

T: How many singles fit into a flat?

$\mathrm{C}$ : A hundred.

T: How many singles fit into a cube?

C: A thousand.

T: How many of the longs fit into the cube?

C: [Picks up long, holds vertically, and places on top of three adjacent squares on the top face of the cube in succession] A hundred.

T: How did you do that?

C: Since there's a hundred here [taps top face of cube] you just go 1, 2, 3, 4 [moving the vertically aligned long from near to far along the rightmost column of the top face of the cube]. Then you just keep on filling them in [places vertically aligned long in center of the top face]. If you take this off [top face] and stick like, a hundred of them in [gestures inserting beginning on left column, from near to far].

In responding to the final task, Cody seemed to accept each single in the visible face of the cube as representing a long that went ten singles deep into the cube. He still relied on figurative material to coordinate the third level of units (the number of singles in the visible face), but he no longer conflated units and was able to mentally create a long for each single, indicating that he had interiorized a two-level structure for coordinating the 100 singles as 100 10s (longs).

Episodes 14a and 14b: October 16 and November 27. This second pair of contrasting episodes pertains to a continuous context, in which Cody would not be able to visualize discrete units within a composite unit. These tasks involved measuring off volumes of water with measuring spoons and measuring cups. The first episode (Episode 14a) occurred during the $9^{\text {th }}$ teaching session. Cody was given a clear plastic $1 / 2$ cup, a black plastic $1 / 4$ cup, a silver tablespoon [1/16 cup], and a yellow plastic teaspoon [1/48 cup]. Cody shared that he was familiar with the use of measuring spoons/cups when making French toast at home. After a brief discussion about the kinds of questions the teacher-researcher might ask, the teacher-researcher asked Cody to 
measure off water in order to determine the relationships between teaspoons and tablespoons, between tablespoons and $1 / 4$ cups, and between $1 / 4$ cups and $1 / 2$ cups. Cody did this and recorded information, as illustrated in Figure 6.

$$
\begin{aligned}
& \text { y tsp fills a stosp otimes } \\
& \text { three yellow tea spouns fill I siver fabtespoon }
\end{aligned}
$$

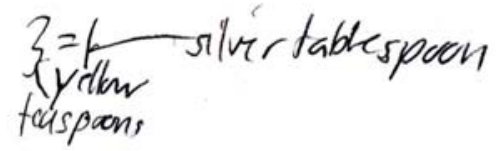

$$
\begin{aligned}
& \text { 3.y.tsp }=1 \text { s. tbsp } \\
& 4 \text { sittep }=1 \text { ib. cup } \\
& 2 b_{1} \cdot a_{p}=1 \text { cieup }
\end{aligned}
$$

Figure 6. Cody's notes on measuring spoons/cups.

The following protocol began soon after Cody had recorded his notes.

T: Do you know how many teaspoons go into a tablespoon?

C: [Looks at paper] 3.

T: Do you know how many tablespoons go into the small black cup?

C: 4 [looking at paper].

T: Do you know how many - all right, so you have that information. What I'm claiming is that you have enough information on your sheet that you can figure out all the other ones. After you figure them out, you can check them. So you'll be allowed to check them with the water later. If you want to draw any pictures, too, you're allowed to draw pictures. Are you ready for the questions?

C: Yeah.

T: Okay. First question is, how many teaspoons are there in a tablespoon?

C: [looking at paper] 3.

T: Okay. Second question is, how many teaspoons - how many yellow teaspoons - are there in small cup - a small black cup?

C: [Looks at paper at first, then looks at the pan containing the objects.] 
T: How many yellow teaspoons [holds up] are in the small black cup [holds up].

C: About - five.

$\mathrm{T}$ : Can you figure out, from your relationships?

$\mathrm{C}$ : Because, a tablespoon is bigger.

T: Okay.

C: So, five would probably be right, 5 or 6 [looking at the objects].

T: So, what did you know about the tablespoon?

C: [Looks at paper, then looks at teacher-researcher] It's bigger, and there's 4 of them, I

think it would take like 5 .

Note that, in answering questions about units with a direct relationship, Cody had consistently referred to his notes. But despite the fact that he had just finished recalling the relationship between teaspoons and tablespoons and the relationship between tablespoons and $1 / 4$ cups, he only made use of the latter in estimating the relationship between teaspoons and $1 / 4$ cups. In order words, the task involved an intermediary unit of tablespoons, but Cody seemed unable to leverage that unit as part of a three-level relationship. Rather, he simply reasoned that there would be more teaspoons than tablespoons in a $1 / 4$ cup because tablespoons are bigger, and his reasoning seemed additive, rather than multiplicative, in that he added just one more teaspoon to the known number of tablespoons. Episode 14b, which occurred during the $14^{\text {th }}$ (and final) teaching session, demonstrates marginal growth and persistent problems for Cody when working with this continuous quantity.

T: All right. First question is, how many yellow teaspoons are in a silver tablespoon.

C: [Glancing down at paper] 3.

$\mathrm{T}$ : How many silver tablespoons are in a small black cup?

C: 1 - I mean, 4.

T: How many small black cups are in the bigger yell- uh, clear cup.

C: Uh, 2.

$\mathrm{T}$ : Let me ask you again - how many teaspoons are in a tablespoon?

C: How many teaspoons... [looks at sheet] 3.

$\mathrm{T}$ : How many teaspoons are in a small black cup?

$\mathrm{C}$ : [12 second pause, looking at paper, resting head on arms] $6 \mathrm{I}$ think.

T: How'd you do that?

$\mathrm{C}$ : Because, there's be 4 in here [points at second line in paper about the tablespoon and quarter cup] and that's [picks up the two measuring spoons]. That's bigger - it's like twice as big as it, so, I thought it was like, 1 tablespoon, so this would go into here [picks 
up tablespoon and holds it over the quarter cup, looks at paper] 4 times, and 4 plus 3 would be 7 . So it would be 7 .

Here, we see some indication that Cody was attempting to establish a multiplicative relationship ("twice as big") and to coordinate three levels of units, including the relationship between teaspoons and tablespoons and the relationship between tablespoons and $1 / 4$ cups. However, Cody failed to establish a multiplicative relationship between these units and simply added the two numbers in the relationships instead. Although his actions might demonstrate limitations in the generalizability of his new ways of operating, they can also be seen as consistent with those new ways of operating. Specifically, Cody consistently relied on repeated addition to establish multiplicative relationships and he often relied on figurative material to keep track of his iterations; when working with continuous quantities, there are no figurative cues for him to do so.

Summary. During the final four teaching sessions, Cody exhibited markedly different ways of operating with units. Chief among these, Cody seemed to have interiorized a two-level structure for assimilating with composite units and had internalized actions for coordinating three levels of units. Both of these developments provide indication that Cody was operating at Stage 2. Moreover, Cody's growth generalized so that he could operate similarly in other discrete contexts. On the other hand, Cody's growth in the continuous context was more muted, presumably because the figurative material in that context did not help him distinguish the three levels of embedded units that he needed to internalize.

\section{Conclusions}

Units coordination plays a critical role in students' abilities to meaningfully engage in a 
large swath of the middle school curriculum, including fractions, integer addition, and algebraic reasoning (Ellis, 2007; Hackenberg \& Tillema, 2009; Izsák, Jacobsen, de Araujo, \& Orrill, 2012; Norton \& Wilkins, 2012; Olive \& Çağlayan, 2008; Steffe \& Olive, 2010; Ulrich, 2012). Students operating at Stage 1 experience substantial constraints as they attempt to assimilate and resolve problematic situations in these domains (Hackenberg, 2013; Hackenberg \& Tillema, 2009;

Steffe, 1992). Our approach to provoking Cody's construction of a two-level assimilatory structure was an attempt to take on these constraints as an instructional problem. This approach was informed by previous research on units coordination and related findings from neuroscience. Namely, our approach involved (1) challenging Cody to simultaneously keep in mind multiple embedded relationships (Steffe, 1992); (2) providing him with physical manipulatives to enact and keep track of relationships between various units (Clements, 2000); and (3) prompting him to reflect on the actions he used to build those relationships (Wheatley, 1992).

We have provided evidence that our approach supported growth in Cody's units coordinating activity, from Stage 1 to Stage 2. In this concluding section, we characterize Cody's growth, along with constraints experienced by the teacher-research in attempting to provoke such growth. Then we consider the results of our approach more generally, within our neo-Piagetian and cognitive neuroscience framework. Finally, we speculate on implications for instruction and future research.

\section{Characterizing Growth and Constraints within the Teaching Experiment}

Cody's actions at the beginning of the teaching experiment indicated that he had to build up composite units from smaller units — characteristic of Stage 1. In line with our approach, we engaged Cody in tasks that allowed him to rely on figurative material to work with more than two levels of units. He often conflated these units, but this may be a sign of progress in itself 
because it indicates an attempt to attend to all three levels (Sophian, 2004). By the end of the teaching experiment he seemed to have internalized this activity so that he no longer needed the figurative material to carry it out—characteristic of Stage 2.

Despite his gains, we recognized continued limitations in Cody's ways of operating with units, even at the end of the teaching experiment. Chief among these limitations, Cody did not seem able to coordinate units in continuous contexts in the same way he could in discrete contexts. For most of the teaching sessions, the teacher-researcher supplied Cody with discrete figurative material (e.g., chips and base-10 blocks) on which he could rely to coordinate three levels of units in activity. By the end of the teaching experiment, Cody had internalized this activity so that he could apply it across discrete contexts without reliance on the figurative material. However, this internalized activity did not seem to generalize to continuous contexts.

We conjecture that the limitation is due to the lack of physical referents for the embedded units within composite units that are continuous. For example, a tablespoon contains three teaspoons, but these three units are not as evident within the tablespoon as they would be with three chips within a cup. Rather, students have to produce the units within a continuous composite unit through some kind of segmenting or partitioning activity (Steffe, 1991), which involves breaking down the composite unit. As we have seen, Cody tended to build up composite units from the smallest unit. Although he learned to reverse that activity by the end of the teaching experiment, he might not have interiorized it. This would involve organizing his partitioning and iterating operations as inverses within an assimilatory structure called splitting (Steffe, 2002). Splitting is in the purview of Stage 2 students, but it requires further development (Steffe \& Olive, 2010).

\section{Sensorimotor, Internalized, and Interiorized Activity}


Piaget (1972/1970) distinguished several stages in children's development, from sensorimotor activity to formal operational thought. Neo-Piagetian perspectives often question the strict age-based delineations Piaget made between these stages but, nonetheless, value their contributions in explaining logico-mathematical development (Fischer \& Bidell, 2006; PascualLeone, Johnson, \& Agostino, 2010). Here, we have described stages in students' development of units coordination, relating them to both Piaget's developmental psychology and findings from neuroscience.

The basic idea we took from Piaget (1972/1970) is that students can perform coordinations in activity (physical or imagined) before interiorizing those coordinations as assimilatory structures. This distinction fits distinctions in neuroscience between (1) physical actions, which correlate with neural activity in the sensorimotor region of the neo-cortex; (2) imagined (internalized) actions, which correlate with neural activity in the premotor region within the frontal lobe; and (3) automated numeric/spatial actions, which correlate with neural activity in the parietal lobe (Cohen et al., 1996; Piazza, Izard, Pinel, Le Bihan, \& Dehaene, 2004). Additionally, our approach was informed by the neuroscience finding that mathematical development correlates with a shift in neural activity from frontal to parietal, and that frontalparietal coherence — manifested in working memory — might support this shift (Logie, 1995; Rivera, Reiss, Eckert, \& Menon, 2005; Sauseng, Klimesch, Schabus, \& Doppelmayr, 2005). We engaged Cody in a sequence of tasks designed to elicit multiple two-level units coordinations, in activity, with the idea that demands on working memory would induce the kind of frontal-parietal coherence that would support reorganizations in his operational schemes to form a (two-level, assimilatory) units coordinating structure. By the fifth session (Episode 8) we had already seen evidence of the effectiveness of our approach: Having built up a two-level unit 
through activity, this composite unit was still available to him (presumably in working memory) during the task that immediately followed so that he could break it down again—something he had been unable to do in earlier episodes. This ability to build up and break down a composite unit demonstrates the kind of reversibility Piaget (1970/1968) attributed to operational structures. By Episode 12, Cody could break down composite units using multiplicative relationships even before building up those relationships through activity.

In cases where Cody had to work across three or more levels of units, he was able to rely on figurative material (manipulatives, such as chips and cups, and drawings) to connect the composite units he had produced. Reflecting on new ways of acting may have helped him to take this activity as an object of thought (Wheatley, 1992), thereby supporting the construction of actions on objects that characterize activity within the parietal lobe (Buccino et al., 2001). By the end of the teaching experiment Cody demonstrated that he had internalized the activity of coordinating three levels of units, no longer requiring figurative material. We take this as further indication that Cody had interiorized a two-level structure and was able to act on that structure as an object.

\section{Instructional Implications}

There is one other well-documented case of stage-wise growth in units coordination as a result of instructional interaction, and that case has several similarities to Cody's case. Steffe (1991) had been working with a first-grade student, named Tyrone, who was operating at Stage 0 (Tyrone had not yet interiorized even one level of units and had to rely on sensorimotor activity in order to count). As in our approach with Cody, Steffe supported Tyrone's interiorization of units by providing him with figurative material on which to act (counters) and by prompting Tyrone to reflect on his actions. Also similar to our approach, Tyrone's growth occurred through 
a sequence of cognitively demanding tasks, followed by a prolonged period of rest.

Steffe attributed Tyrone's growth to a metamorphic accommodation - a reorganization of actions (in this case, his internalized counting acts) as objects for acting upon. This reorganization took a prolonged period of time but was set in motion by provocations in which Steffe had challenged Tyrone to determine the total number of counters across two hidden collections. For example, Steffe told Tyrone there were eight counters under one cloth and five counters under another. Tyrone solved the task by pointing to the first cloth eight times while counting and then continuing to count while imagining the figurative pattern for 5 . This demanding activity—counting the entire collection while keeping track of counts within the pattern—seemed to induce counting on, which coincides with Stage 1.

Similarities between Cody's case and Tyrone's case emphasize the importance of maintaining the cognitive demand of tasks (Henningsen \& Stein, 1997). The units coordination framework helps explain how to appropriately design cognitively demanding tasks. Although they followed similar guidelines, the tasks we posed to Cody differed considerably from those Steffe posed to Tyrone. This is because, in each case, we needed to engage students in coordinating $n+1$ levels of units in activity, where $n$ was determined by stages of units coordination; Cody had been operating at Stage 1, and Tyrone had been operating at Stage 0 .

The neuroscience framework helps explain how appropriately demanding tasks can promote the kinds of reorganizations (metamorphic accommodations) that constitute stage-wise growth. Lack of sustained cognitive demand may explain the scarcity of cases like Tyrone's and Cody's and the intransigence of units coordination stages (Steffe \& Cobb, 1988; Steffe \& Olive, 2010). Furthermore, given the role units coordination plays across mathematical domains, it may explain some of the stubbornness teachers experience in students' development of algebraic 
reasoning, multiplicative reasoning, and fractions (National Mathematics Advisory Panel, 2008).

\section{Implications for Future Research}

We have offered a single case of stage-wise development of units coordination, from Stage 1 to Stage 2 (Cody's case), and we have noted parallels between that development and the stage-wise development from Stage 0 to Stage 1 (Tyrone's case). Future research might build additional cases so that we draw generalizations across them. Does the approach outlined here support students' development of units coordination in general? What modifications need to be made to support units coordination in continuous contexts? Future research might also elucidate the role of cognitive demand and working memory in mathematical development. From a neurological perspective, we might ask how sustained cognitive demand can support the kinds of frontal to parietal shifts in activity that correspond to the interiorization of mathematical structures.

\section{Acknowledgements}

This work was supported by the National Science Foundation (NSF) under Grant No. DRL-1118571, and the Institute for Society, Culture and Environment (ISCE) at Virginia Tech. Any opinions, findings, and conclusions or recommendations expressed in this material are those of the authors and do not necessarily reflect the views of NSF or ISCE.

\section{References}

Ansari, D., \& Dhital, B. (2006). Age-related changes in the activation of the intraparietal sulcus during nonsymbolic magnitude processing: An event-related functional magnetic resonance imaging study. Journal of Cognitive Neuroscience, 18(11), 1820-1828. 
Behr, M. J., Lesh, R., Post, T. R., \& Silver, E. A. (1983). Rational number concepts. In. R. Lesh \& M. Landau (Eds.), Acquisition of mathematics concepts and processes, (pp. 91-126). New York: Academic Press.

Blanton, M. L. \& Kaput, J. J. (2005). Characterizing a classroom practice that promotes algebraic reasoning. Journal for Research in Mathematics Education, 36(5), 412-446.

Boyce, S., \& Norton, A. (in review). Simultaneity in the Construction of Units Coordination Structures. Journal for Research in Mathematics Education.

Chochon, F., Cohen, L., Van De Moortele, P. F., \& Dehaene, S. (1999). Differential contributions of the left and right inferior parietal lobules to number processing. Journal of Cognitive Neuroscience, 11(6), 617-630.

Clement, J., Lochhead, J., \& Monk, G. S. (1981). Translation difficulties in learning mathematics. American Mathematical Monthly, 286-290.

Clements, D. H. (2000). Concrete Manipulatives, Concrete Ideas. Contemporary Issues in Early Childhood, 1(1), 45-60.

Cohen, M. S., Kosslyn, S. M., Breiter, H. C., DiGirolamo, G. J., Thompson, W. L., Anderson, A. K., .. \& Belliveau, J. W. (1996). Changes in cortical activity during mental rotation: A mapping study using functional MRI. Brain, 119(1), 89-100.

Confrey, J. (1990). A review of the research on student conceptions in mathematics, science, and programming. Review of Research in Education, 3-56.

Delazer, M., Ischebeck, A., Domahs, F., Zamarian, L., Koppelstaetter, F., Siedentopf, C. M., ... \& Felber, S. (2005). Learning by strategies and learning by drill—Evidence from an fMRI study. Neuroimage, 25(3), 838-849. 
Ellis, A. B. (2007). The influence of reasoning with emergent quantities on students' generalizations. Cognition and Instruction, 25, $439-478$.

Fischbein, E., Deri, M., Nello, M. S., \& Marino, M. S. (1985). The role of implicit models in solving verbal problems in multiplication and division. Journal for Research in Mathematics Education, 3-17.

Fischer, K. W., \& Bidell, T. R. (2006). Dynamic development of action, thought, and emotion. In W. Damon \& R. M. Lerner (Eds.), Theoretical models of human development. Handbook of child psychology (6th ed., Vol. 1, pp. 313-399). New York: Wiley.

Hackenberg, A. J. (2010). Students' reasoning with reversible multiplicative relationships. Cognition and Instruction, 28(4), 383-432.

Hackenberg, A. J. (2013). The fractional knowledge and algebraic reasoning of students with the first multiplicative concept. The Journal of Mathematical Behavior, 32(3), 538-563.

Hackenberg, A. J., \& Tillema, E. S. (2009). Students' whole number multiplicative concepts: A critical constructive resource for fraction composition schemes. The Journal of Mathematical Behavior, 28(1), 1-18.

Henningsen, M., \& Stein, M. K. (1997). Mathematical tasks and student cognition: Classroombased factors that support and inhibit high-level mathematical thinking and reasoning. Journal for Research in Mathematics Education, 524-549.

Izsák, A., Jacobsen, E., de Araujo, Z., \& Orrill, C. H. (2012) Measuring mathematical knowledge for teaching fractions with drawn quantities. Journal for Research in Mathematics Education, 43(4). 
Kaput, J. J. (1989). Supporting Concrete Visual Thinking in Multiplicative Reasoning:

Difficulties and Opportunities. Focus on Learning Problems in Mathematics, 11(1), 3547.

Kaput, J. and Clement, J. (1979). Letter to the editor. Journal of Children's Mathematics Behavior, 2(2), p. 208

Kieren, T. E (1979). The development in children and adolescents of the construct of rational numbers as operators. Alberta Journal of Educational Research, 25(4), 234-47.

Lamon, S. J. (2007). Rational numbers and proportional reasoning: Toward a theoretical framework for research. In F. K. Lester (Ed.), Second handbook of research on mathematics teaching and learning, (pp. 629-667). Charlotte, NC: Information Age Publishing.

Logie, R. H. (1995). Visuo-spatial working memory. Hove, UK: Lawrence Erlbaum Associates. National Mathematics Advisory Panel. (2008). Report of the task group on conceptual skills and knowledge. Washington, DC: U.S. Department of Education.

Nemirovsky, R., \& Ferrara, F. (2009). Mathematical imagination and embodied cognition. Educational Studies in Mathematics, 70, 159-174.

Norton, A., \& D'Ambrosio, B. S. (2008). ZPC and ZPD: Zones of teaching and learning. Journal for Research in Mathematics Education 39, 220-246.

Norton, A., \& Wilkins, J. L. M. (2012). The splitting group. Journal for Research in Mathematics Education, 43(5), 557 - 583.

Olive, J. \& Çağlayan, G. (2008). Learner's difficulties with quantitative units in algebraic word problems and the teacher's interpretations of those difficulties. International Journal of Science and Mathematics Education, 6, 269-292. 
Olive, J., \& Vomvoridi, E. (2006). Making sense of instruction on fractions when a student lacks necessary fractional schemes: The case of Tim. The Journal of Mathematical Behavior 25(1), 18-45.

Pascual-Leone, J., Johnson, J., \& Agostino, A. (2010). Mental attention, multiplicative structures, and the causal problems of cognitive development. In The Developmental Relations among Mind, Brain and Education (pp. 49-82). Springer Netherlands.

Piaget, J. (1970). Structuralism (C. Maschler, Trans.). New York: Basic Books (Original work published 1968).

Piaget, J. (1972). The principles of Genetic Epistemology (W. Mays trans.) New York: Basic Books (Original work published, 1970).

Piazza, M., Izard, V., Pinel, P., Le Bihan, D., \& Dehaene, S. (2004). Tuning curves for approximate numerosity in the human intraparietal sulcus. Neuron, 44(3), 547-555.

Rivera, S. M., Reiss, A. L., Eckert, M. A., \& Menon, V. (2005). Developmental changes in mental arithmetic: evidence for increased functional specialization in the left inferior parietal cortex. Cerebral Cortex, 15(11), 1779-1790.

Sauseng, P., Klimesch, W., Schabus, M., \& Doppelmayr, M. (2005). Fronto-parietal EEG coherence in theta and upper alpha reflect central executive functions of working memory. International Journal of Psychophysiology, 57, 97-103.

Simon, M. A., \& Tzur, R. (2004). Explicating the role of mathematical tasks in conceptual learning: An elaboration of the hypothetical learning trajectory. Mathematical Thinking and Learning, 6(2), $91-104$. 
Sophian, C. (2004). A prospective developmental perspective on early mathematics instruction. Engaging young children in mathematics: Standards for early childhood mathematics education, 253-266.

Steffe, L. P. (1991). Operations that generate quantity. Learning and Individual Differences, 3(1), 61-82.

Steffe, L. P. (1992). Schemes of action and operation involving composite units. Learning and Individual Differences, 4(3), 259-309.

Steffe, L. P. (1994). Children's Multiplying Schemes. In G. Harel \& J. Confrey (Eds.), The development of multiplicative reasoning in the learning of mathematics (pp. 3-39). Albany, NY: SUNY Press.

Steffe, L. P. (2002). A new hypothesis concerning children's fractional knowledge. Journal of Mathematical Behavior, 20(3), 267-307.

Steffe, L. P., \& Cobb, P. (1988). Construction of arithmetical meanings and strategies. New York, NY: Springer.

Steffe, L. P., \& Olive, J. (2010). Children's fractional knowledge. NY: Springer.

Steffe, L. P., \& Thompson, P. W. (2000). Teaching experiment methodology: Underlying principles and essential elements. In A. E. Kelly \& R. A. Lesh (Eds.), Handbook of research design in mathematics and science education, (pp.267-306). Mahwah, NJ: Lawrence Erlbaum Associates.

Ulrich, C. L. (2012). Additive relationships and signed quantities (Doctoral dissertation). Retrieved from http://www.libs.uga.edu/etd/

Ulrich, C. (in press). Stages in constructing and coordinating units additively and multiplicatively (Part 1). For the Learning of Mathematics, 35(3). 
Varma, S., \& Schwartz, D. L. (2008). How should educational neuroscience conceptualise the relation between cognition and brain function? Mathematical reasoning as a network process. Educational Research, 50(2), 149-161.

von Glasersfeld, E. (1995). A constructivist approach to teaching. In L. P. Steffe, \& J. Gale (Eds.), Constructivism in education (pp. 3- 15). Hillsdale, NJ: Erlbaum.

von Glasersfeld, E., \& Steffe, L. P. (1991). Conceptual models in educational research and practice. The Journal of Educational Thought, 25(2), 91 - 102.

Wheatley, G. H. (1992). The role of reflection in mathematics learning. Educational Studies in Mathematics, 23(5), 529-541. 